\title{
Protospirura numidica criceticola, n. subsp. parasite de Rongeurs Cricetidae du Brésil
}

\author{
CYCLE EVOLUTIF
}

\author{
par J.-C. QUENTIN, Y. KARIMI et C. RODRIGUEZ DE ALMEIDA \\ [Laboratoire de Zoologie (Vers) associé au C.N.R.S., \\ Muséum National d'Histoire naturelle, Paris]
}

\section{Résumé}

Un Spiruride du groupe Protospirura numidica Seurat, 1914, a été récolté fréquemment chez Zygodontomys lasiurus (Lund, 1839) et, rarement, chez Calomys callosus (Rengger, 1830) (Rongeurs Cricetidae), à Exu, Pernambuco, Brésil.

Compte tenu des différences géographiques, écologiques, et de la longueur plus importante des spicules chez le mâle, les auteurs considèrent que ce Nématode appartient à une sous-espèce distincte de l'espèce de Seurat. Ils proposent donc de nommer cette sous-espèce : $P$. numidica criceticola $n$. subsp., et y rattachent les Helminthes jusqu’à présent nommés Protospirura numidica en Amérique du Nord.

Le cycle biologique et les différents stades larvaires de cet Helminthe sont étudiés expérimentalement chez l'hôte intermédiaire Doru lineare Escherich, et chez des hôtes définitifs: Rongeurs Cricetidae appartenant aux espèces : $Z$. lasiurus (Lund, 1839), Calomys callosus (Rengger, 1830), Oryzomys nigripes (Desmarest, 1819), et un Rongeur Muridae africain: Hybomys univittatus Peters.

La présence de $P$. numidica criceticola chez les Rongeurs Zygodontomys semble résulter de facteurs écologiques.

\section{Summary}

A Spiruride from the group Protospirura numidica Seurat, 1914 was frequently collected in Zygodontomys lasiurus (Lund, 1839) and rarely in Calomys callosus (Rengger, 1830) (Cricetidae) in Exu, Pernambuco, Brazil. 
Regarding the geographical and ecological differences, and the larger spicules in the male, the authors consider this nematod to belong to a distinct subspecies of the species of Seurat. In consequence, the name $P$. numidica criceticola $\mathrm{n}$. subsp. is proposed and includes all the North american Helminths named up to now Protospirura numidica.

The life cycle and the different larval stages of this Helminth are described in the intermediate host Doru lineare and in the definitive hosts: Cricetidae Rodents belonging to the species: Z. lasiurus (Lund, 1839), Calomys callosus (Rengger, 1830), Oryzomys nigripes (Desmarest, 1819), and an African Muridae : Hybomys univittatus Peters.

The presence of Protospirura numidica criceticola in the Rodents Zygodontomys seems to be the resultant of ecological factors.

HôTES, LOCALITÉ, DATES DE RÉCOLTE : au cours des autopsies effectuées sur des Rongeurs Cricetidae : 225 Zygodontomys lasiurus (Lund, 1839), 22 Calomys callosus (Rengger, 1830) à Exu, Pernambuco, Brésil, durant les mois de novembre, décembre 1966, et les mois de mai, juin, juillet 1967, 33 mâles et 60 femelles ont été récoltés dans l'estomac des Zygodontomys, 3 femelles seulement ohez les Calomys.

Les types de cette sous-espèce ont été recueillis chez un Zygodontomys piégé au sitio Serra Sapo, Exu, le 3-6-67, $\mathrm{n}^{\circ} 139$ u.

\section{Description.}

La bouche, chez le mâle et la femelle, est pourvue de deux grandes pseudo-lèvres latérales trilobées, chaque lèvre étant ornée de dents sur son bord interne (fig. $1 \mathrm{~B}$ ) presque semblables à celles observées sur un spécimen femelle paratype de Protospirura numidica Seurat, 1914. Le plateau céphalique porte huit papilles céphaliques du cycle externe, groupées deux par deux, et deux amphides. La cavité buccale est bien développée.

1) Mâle holotype: Sa longueur est de $24,5 \mathrm{~mm}$, sa largeur est de $600 \mu$; les deirides, l'anneau nerveux, le pore excréteur sont respectivement situés à 250,380 et $530 \mu$ de l'apex. Le pharynx, de section circulaire, mesure $120 \mu$. L'œsophage musculaire est long de $370 \mu$, l'œsophage glandulaire de $3850 \mu$. Les spicules sont très dissymétriques, le droit mesure $1250 \mu$ de long, sa largeur est de $30 \mu$; le gauche est long de $470 \mu$, large de $75 \mu$. Le gubernaculum mesure $160 \mu$. Les longueurs respectives des spicules droits et gauches des trois autres mâles récoltés chez le même Rongeur sont : 1260 et $500 \mu, 1130$ et $480 \mu, 1380$ et $470 \mu$.

La queue mesure $330 \mu$. Les papilles pré-anales énormes et sessiles sont au nombre de quatre paires (fig. 1 C) ; une papille impaire se trouve sur le bord antérieur du cloaque. En arrière du cloaque sont disposées deux paires de grosses papilles, cinq paires de petites et une paire d'amphides, toutes très apparentes. 

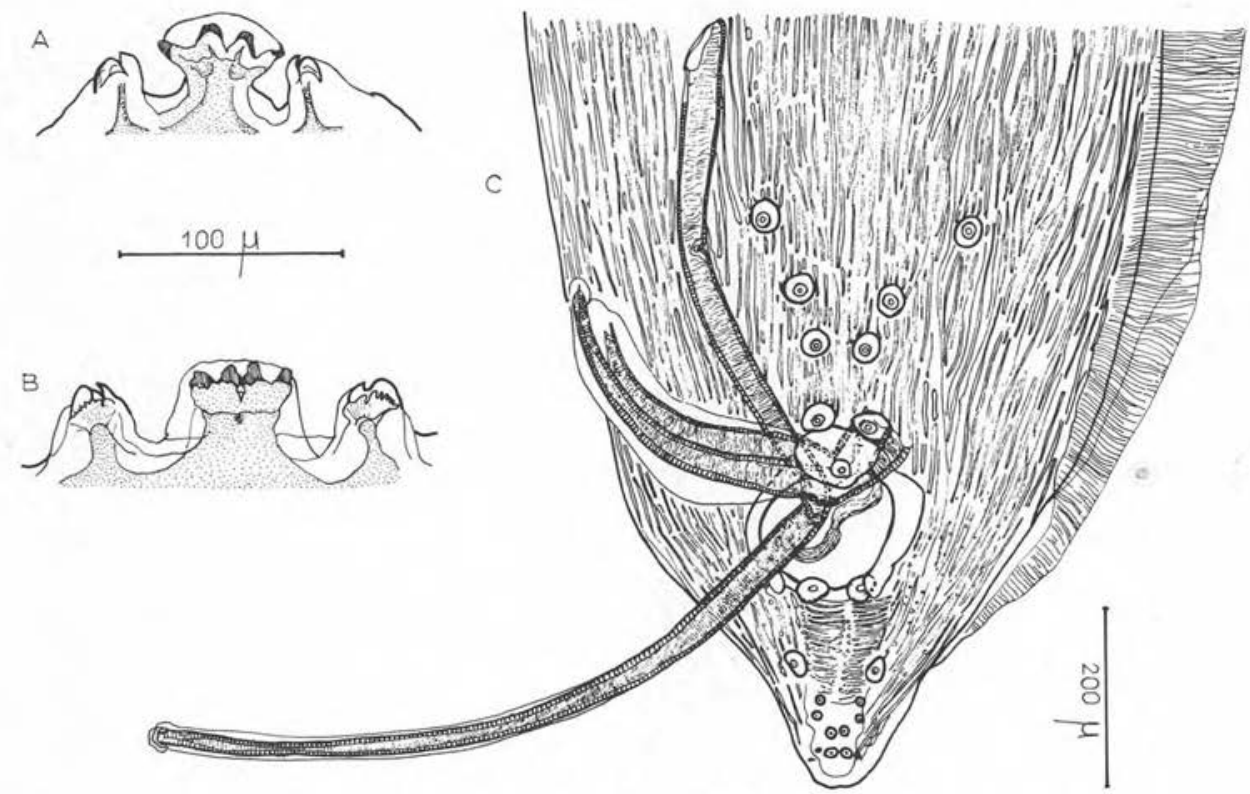

Fig. 1. - Adultes. - A : vue interne de la pseudo-lèvre latérale d'un spécimen $\subsetneq$ paratype de Protospirura numidica Seurat, $1914-$ B $:$ idem., $q$ de $P$. numidica criceticola parasite du Zygodontomys lasiurus (Lund, 1839) - C : vue ventrale de la bourse caudale d'un mâle de $P$. numidica criceticola parasite de Zygodontomys. A, B : éch. $100 \mu$. C: éch. $200 \mu$

2) Femelle allotype: La femelle mesure $50 \mathrm{~mm}$ de long sur $760 \mu$ de large, les deirides, l'anneau nerveux et le pore excréteur sont respectivement situés à 265 , 460 et $570 \mu$. Le pharynx mesure $170 \mu$, l'œsophage musculaire $370 \mu$, l'œsophage glandulaire $5600 \mu$. La vulve est située à $21 \mathrm{~mm}$ de la pointe caudale. L'ovéjecteur est long de $1540 \mu$ et contient des œufs embryonnés dont les dimensions sont $45 \times 33 \mu$.

\section{Discussion.}

Ces Helminthes correspondent par leur morphologie générale, le dessin de la face interne des pseudo-lèvres, celui de la disposition des papilles cloacales chez les mâles, à la description et aux dessins donnés par Seurat de Protospirura numidica Seurat, 1914. Les types ont été récoltés et décrits en Afrique du Nord chez le Chat ganté Felis ocreata Gmel., puis Seurat (1916) a retrouvé l'espèce chez Arvicanthis barbarus L., qu'il a considéré comme l'hôte normal. Chez cet hôte, Seurat ne donne pour toute dimension que la longueur de la queue : $210 \mu$.

$\mathrm{Au}$ Brésil, les mâles que nous avons recueillis, et dont la longueur (20-24,5 mm) est très proche de celle du mâle décrit par Seurat $(22 \mathrm{~mm})$, ont un spicule droit dont la taille, comprise entre 1000 et $1420 \mu$, est toujours supérieure à celle mesurée par Seurat $(830 \mu)$ chez le spécimen type récolté chez Felis ocreata. 
Ce caractère paraît constant et nous pensons qu'il est utile de considérer notre matériel du Brésil comme une sous-espèce distincte de l'espèce de Seurat récoltée en Algérie.

Nous proposons de la nommer Protospirura numidica criceticola n. subsp.

Chitwood, en 1938, donne les dimensions respectives des spécimens étudiés en Algérie par Seurat, et ceux récoltés en différentes régions des Etats-Unis chez Canis latrans et chez des Rongeurs Cricetidae du genre Peromyscus. Les spécimens mâles d'Amérique du Nord ont un spicule droit dont la longueur $(940$ à $1160 \mu)$ reste toujours supérieure à la dimension indiquée par Seurat. Chitwood les nomme $P$. numidica.

Le parasite est signalé de nouveau en Amérique du Nord par Butler et Grundmann en 1957 chez Canis latrans, par Grundmann en 1957 et Grundmann et Frandsen en 1960 chez des Rongeurs Cricetidae du genre Peromyscus. Selon Grundmann, le parasite semble inféodé aux Rongeurs Cricetidae et à un Heteromyidae : Dipodomys ordii.

La faune américaine a donc non seulement des hôtes différents (Cricetidae, Heteromyidae et non Muridae), en outre, une particularité morphologique qui paraît constante (spicule droit plus long). Nous proposons donc de rattacher tous les spécimens américains à la sous-espèce $P$. numidica criceticola $\mathrm{n}$. subsp.

\section{Cycle biologique.}

\section{A. - Développement chez l'Insecte}

1) Matériel. Les Insectes utilisés pour réaliser le cycle évolutif de ce Nématode ont été récoltés durant les mois de juin et juillet 1967 à Exu, dans les champs de maïs fréquentés par les Zygodontomys.

L'évolution des différents stades larvaires a été suivie à une température d'environ $25^{\circ} \mathrm{C}$ chez la Forficule Doru lineare Escherich (1), Insecte qu'il est aisé de se procurer sous les débris végétaux du sol, ou sur la face interne de la ligule des feuilles de maïs, cù il recherche l'ombre durant la période humide de l'année. Durant la période sèche, l'Insecte trouve abri dans le sol dans les courtes galeries creusées par les Rongeurs et Reptiles. Les infestations expérimentales se sont révélées positives chez des Blattes de l'espèce Periblaneta americana Linné (1) vivant dans le même biotone.

Les Insectes pris dans la nature ont été nourris avec une pâte obtenue en écrasant quelques femelles de Protospirura numidica, et en étalant à l'aide d'un pinceau les œufs sur les parois d'un Erlenmeyer, le long desquelles viennent s'accrocher les Forficules et les Blattes.

23 Forficules ont été disséquées pour permettre l'étude des trois premiers stades larvaires et pour obtenir les larves nécessaires à l'infestation de l'hôte définitif : toutes étaient parasitées; le nombre de capsules disséminées dans la cavité abdominale varie, selon les individus, de 2 à 64 .

(1) Nous remercions M. le Professeur Chopard du Muséum National d'Histoire Naturelle qui a bien voulu déterminer les Insectes parasités. 

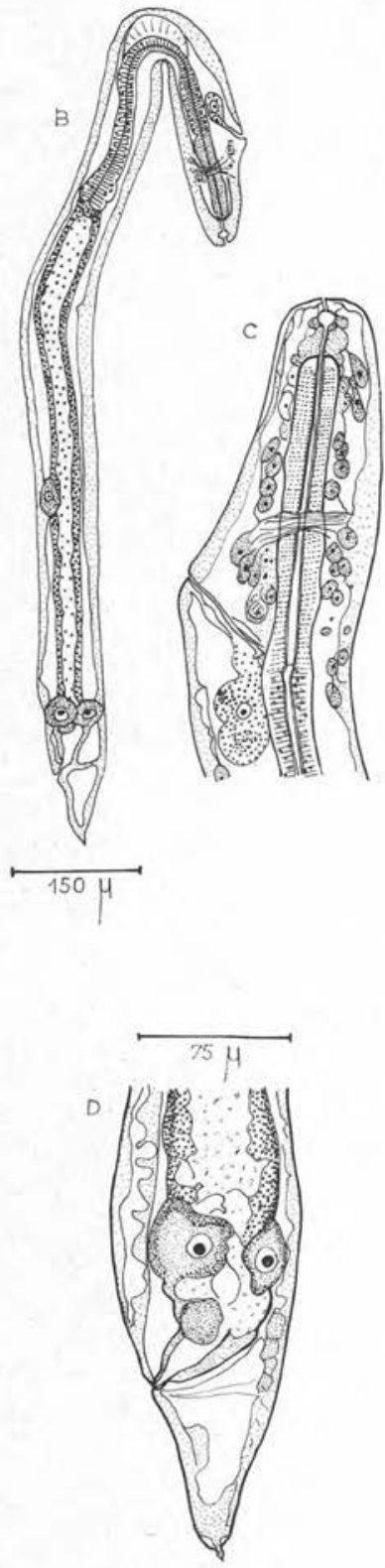
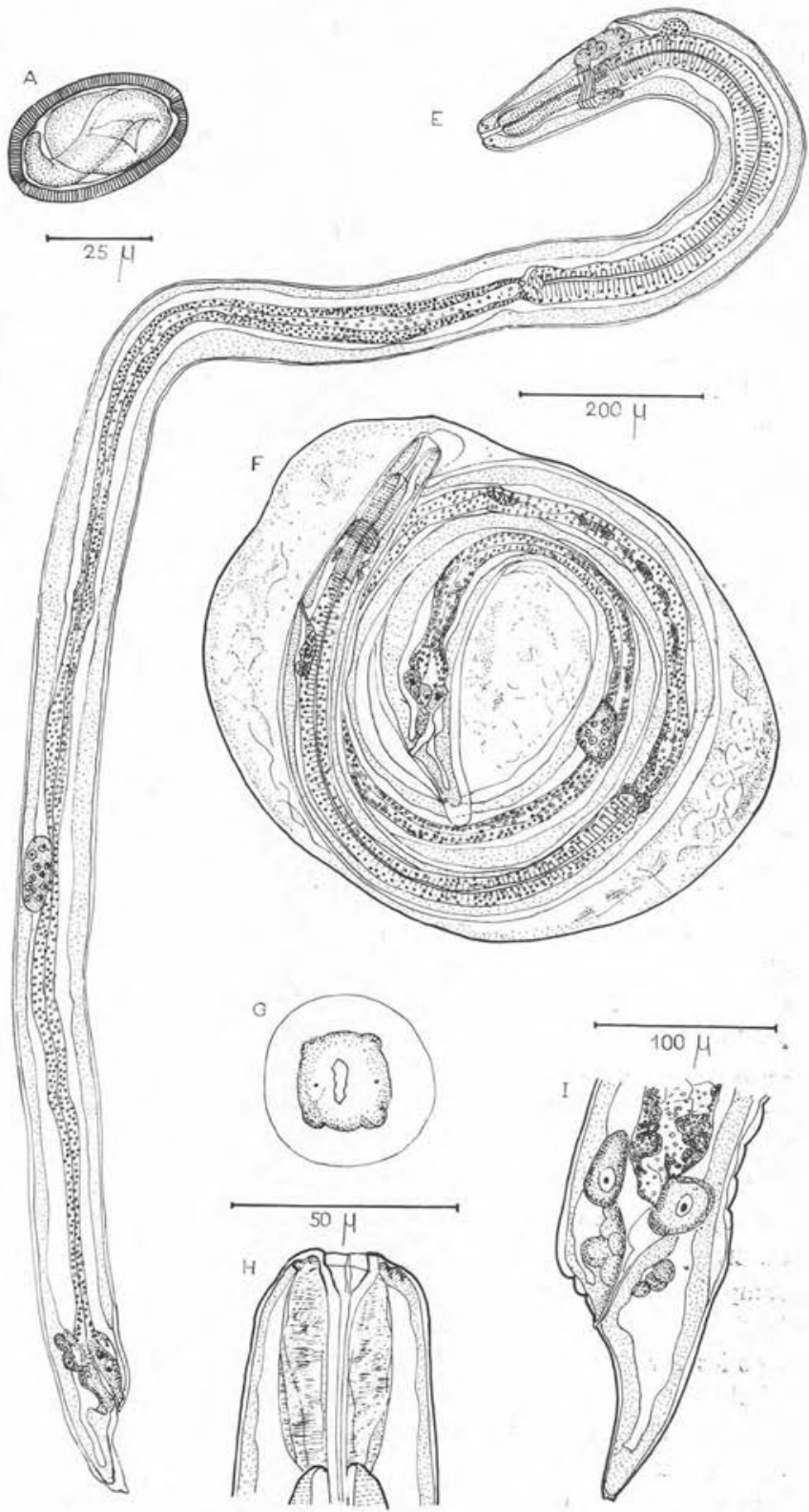

Fig. 2. $1^{\text {er }}$ stade larvaire. - A : Euf embryonné. - B. Larve de 14 jours, fin du $1^{\text {er }}$ stade. C: Extrémité antérieure de cette larve, vue latérale. - D: Extrémité postérieure, vue latérale, $2^{\text {e }}$ stade. - E : larve de 26 jours libérée de sa capsule, vue latérale. $\rightarrow$ F : Larve du $2^{\text {e }}$ stade encapsulée. - $-\mathrm{G}$ : Vue apicale d'une larve du $2^{e}$ stade. $-\mathrm{H}$ : Vue latérale de l'extrémité céphalique. - I : Extrémité postérieure du $2^{e}$ stade larvaire. A : éch. $25 \mu$. B : éch. $150 \mu$. C, D: éch. $75 \mu$. E, F : éch. $200 \mu$. G, H: éch. $50 \mu$. I : éch. $100 \mu$ 
30 Forficules prises simultanément et autopsiées comme témoins ne présentaient pas de capsules dans l'hémocoele.

2) Allure du développement. Les premiers jours de l'infestation, l'observation est rendue difficile par l'abondance des larves de Nématodes Tylenchides qui infestent naturellement ces Insectes. Cependant, il est possible, dès le douzième jour, d'étudier la croissance des capsules dans l'hémocoele, où elles mesurent $350 \mu$ de diamètre. Les capsules atteignent $500 \mu$ de diamètre vers le vingt-sixième jour, et ne dépassent pas $600 \mu$ de diamètre après l'obtention du troisième stade larvaire.

Le premier stade larvaire s'achève vers le quinzième jour où la larve mesure $1000 \mu$ environ. La croissance la plus importante chez l'hôte intermédiaire s'effectue durant le deuxième stade larvaire qui se termine à partir du vingt-sixième jour. La larve mesure $2580 \mu$ de long. Après la deuxième mue, les larves du troisième stade larvaire ont 2800 à $2850 \mu$ de long et atteignent. $3300 \mu$ le trentième jour.

\section{3) MORPHOlOGie Des diffÉRENTS STADES.}

a) Premier stade larvaire (fig. 2 B). La larve, vers la fin du $1^{\text {er }}$ stade (14 jours), mesure $1080 \mu$ de long et $70 \mu$ de large. L'anneau nerveux est situé à $90 \mu$ de l'apex, en avant d'une grosse cellule excrétrice qui s'ouvre à $125 \mu$ de l'apex (fig. $2 \mathrm{C}$ ). Le tube digestif est déjà parfaitement différencié, possédant une partie pharyngienne longue de $23 \mu$, dilatée en son milieu, un œsophage musculaire long de $67 \mu$, et un œsophage glandulaire long de $280 \mu$. L'intestin aboutit à des glandes rectales très volumineuses. L'ébauche génitale, unicellulaire à ce stade, est située à $370 \mu$ de la pointe caudale. La queue est longue de $76 \mu$ et se termine par un mucron (fig. $2 \mathrm{D}$ ).

b) Deuxième stade larvaire. Vers la fin du $2^{\circ}$ stade (fig. $2 \mathrm{E}, \mathrm{F}$ ), les larves présentent au $26^{\circ}$ jour un vestibule buccal bien différencié, profond de $45 \mu$. Une vue apicale de l'extrémité céphalique (fig. $2 \mathrm{G}$ ) montre que l'ouverture buccale préfigure déjà les deux pseudo-lèvres latérales de l'adulte. Les dimensions de cette larve sont: longueur, $2.580 \mu$; largeur, $60 \mu$; l'anneau nerveux et le pore excréteur sont respectivement situés à 125 et $225 \mu$ de l'apex. L'œsophage musculaire mesure $125 \mu$ de long, l'œsophage glandulaire, $650 \mu$. L'ébauche génitale est à $650 \mu$ de la pointe caudale, soit au 1/4 postérieur de la longueur totale. La queue mesure $90 \mu$ de long.

c) Troisième stade larvaire (fig. $3 \mathrm{~A}, \mathrm{~B}$ ). La seconde mue s'effectue aux environs du $27^{\circ}$ jour. La cuticule du $2^{\circ}$ stade qui se décolle de l'extrémité antérieure permet de comprendre la structure de la capsule buccale: long cylindre à parois épaisses appliquées à la face interne de la capsule buccale du $3^{\text {e }}$ stade, profonde de $50 \mu$. Celle-ci présente tous les caractères de l'adulte. L'ouverture buccale est, en effet, fendue en deux pseudo-lèvres trilobées. Le plateau facial porte 4 papilles du cycle externe et 2 amphides, 6 petites papilles du cycle interne sont disposées à l'extrémité de chaque lobe. La larve du $28^{\mathrm{e}}$ jour mesure $2850 \mu$ de long et $110 \mu$ de large. Les deirides bien visibles, l'anneau nerveux et le pore excréteur sont situés respectivement à 100,150 et $230 \mu$ de l'apex, 2 ailes latérales larges de 2,5 $\mu$ naissent en arrière des deirides et se terminent au niveau de l'anus. La longueur de l'œsophage musculaire est de $150 \mu$; celle de l'œsophage glandulaire a considérablement augmenté et atteint $950 \mu$. L'ébauche génitale est située à $700 \mu$ de la pointę caudale. La queue mesure $110 \mu$. La terminaison caudale est remarquable par son ornementation 


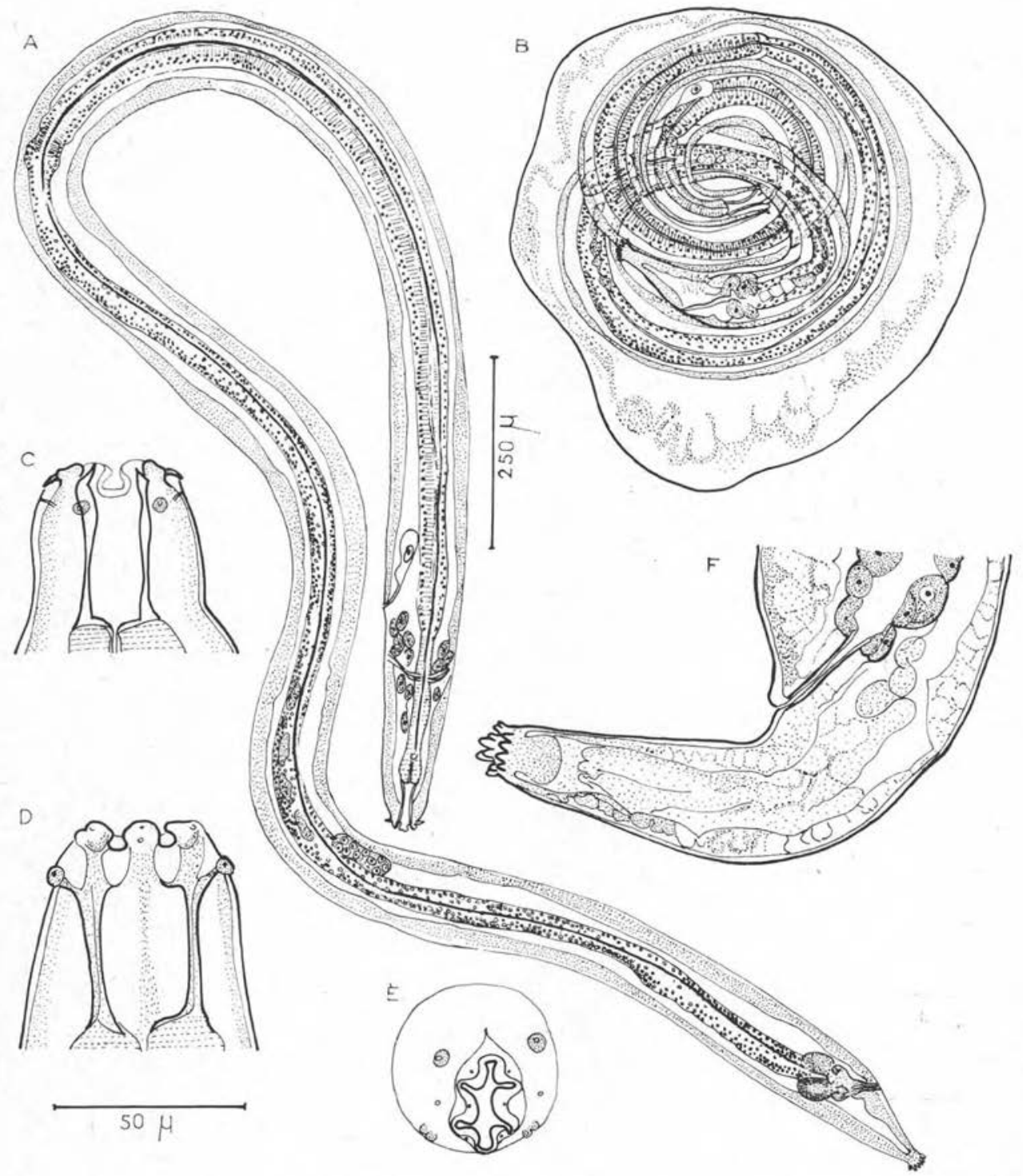

FIG. 3. $3^{\text {e }}$ stade larvaire. - A : Larve de 28 jours libérée de sa capsule, vue latérale. - B : Larve de 28 jours encapsulée. - C : Extrémité céphalique, vue ventrale. - D : idem, vue latérale. E: idem, vue apicale. A, B : éch. $250 \mu$. C, D, E, F : éch. $50 \mu$ 
de petites pointes, au nombre de 12 à 15 , et peut servir à différencier rapidement au binoculaire le $2^{\circ}$ du $3^{e}$ stade larvaire chez l'Insecte, le $3^{\circ}$ du $4^{e}$ stade larvaire chez l'hôte définitif. Les larves du $3^{\circ}$ stade utilisées pour l'infestation mesurent au $30^{\circ}$ jour de leur développement chez l'hôte intermédiaire $3300 \mu$, elles sont larges de 100 à $110 \mu$. Deirides, anneau nerveux et pore excréteur sont situés, respectivement, à 100, 195 et $290 \mu$ de l'apex. Le pharynx mesure $65 \mu$ de long. L'œsophage musculaire atteint $200 \mu$, l'œsophage glandulaire, $1135 \mu$; l'ébauche génitale est à $800 \mu$ de la pointe caudale; la queue mesure $120 \mu$.

\section{B. - Evolution chez l'hôte définitif}

Etant donné la forte infestation de Protospirura numidica criceticola chez l'espèce Zygodontomys lasiurus (Lund, 1839), durant la période sèche de l'année au Brésil, et le très faible pourcentage de ce parasite chez les autres Rongeurs Cricetidae, nous avons cherché à suivre l'évolution des troisième et quatrième stades larvaires et des adultes chez de jeunes Zygodontomys, nés en laboratoire - à comparer cette évolution à celle obtenue par l'infestation expérimentale d'autres Rongeurs Cricetidae : Calomys callosus (Rengger, 1830) et Oryzomys nigripes (Desmarest, 1819), qui dans les conditions naturelles sont peu ou jamais parasités ; - enfin, à savoir si les larves peuvent évoluer chez des Rongeurs très différents d'origine, tels que des Muridae africains.

EXPÉRIMENTATION. Les larves du troisième stade encapsulées, obtenues en disséquant des Forficules infestées vingt-huit à trente jours auparavant, sont placées dans du milieu Vago, puis introduites à l'aide d'une pipette dans la gorge des Rongeurs. Les résultats obtenus sont résumés dans le tableau 1 .

Tableau 1. - Durée d'évolution chez l'hôte définitif (en jours)

\begin{tabular}{|c|c|c|c|c|c|c|c|c|c|c|c|c|c|c|c|c|c|c|}
\hline & & 1 & 5 & 7 & 10 & 11 & 12 & 15 & 16 & 17 & 19 & 20 & 25 & 30 & 33 & 47 & 51 & 55 \\
\hline \multirow{2}{*}{ Z } & a & 6 & 7 & & 10 & & 17 & 2 & 7 & & & 6 & 14 & & 8 & & & 10 \\
\hline & b & 1 & 4 & & 6 & & 10 & 2 & 7 & & & $\begin{array}{l}20 \\
20\end{array}$ & $\begin{array}{l}20^{\circ} \\
4 \%\end{array}$ & & $\begin{array}{l}30 \\
3 \%\end{array}$ & & & 19 \\
\hline \multirow{2}{*}{ C } & $\mathrm{a}$ & & & 15 & & 4 & & & & 7 & 12 & & & & & 15 & & \\
\hline & $\mathrm{b}$ & & & 4 & & 4 & & & & 6 & 3 & & & & & $\begin{array}{l}10 \\
3 \%\end{array}$ & & \\
\hline \multirow{2}{*}{0} & a & & & & & & & & & 7 & & & & 12 & & & 6 & \\
\hline & $\mathrm{b}$ & & & & & & & & & 6 & ${ }^{\circ}$ & & & $\begin{array}{l}20^{\circ} \\
2{ }^{\circ}\end{array}$ & & & $\begin{array}{l}46 \\
19\end{array}$ & \\
\hline & $\begin{array}{l}Z \\
C \\
O\end{array}$ & $C$ & & & & j & & $=\mathrm{n}$ & $\mathrm{mbr}$ & $\begin{array}{l}\text { e de } \\
\text { e de }\end{array}$ & $\begin{array}{l}\text { lar } \\
\text { la }\end{array}$ & $\begin{array}{l}\text { ies } \mathrm{i} \\
\text { es } \mathrm{r}\end{array}$ & estar & & & & & \\
\hline
\end{tabular}




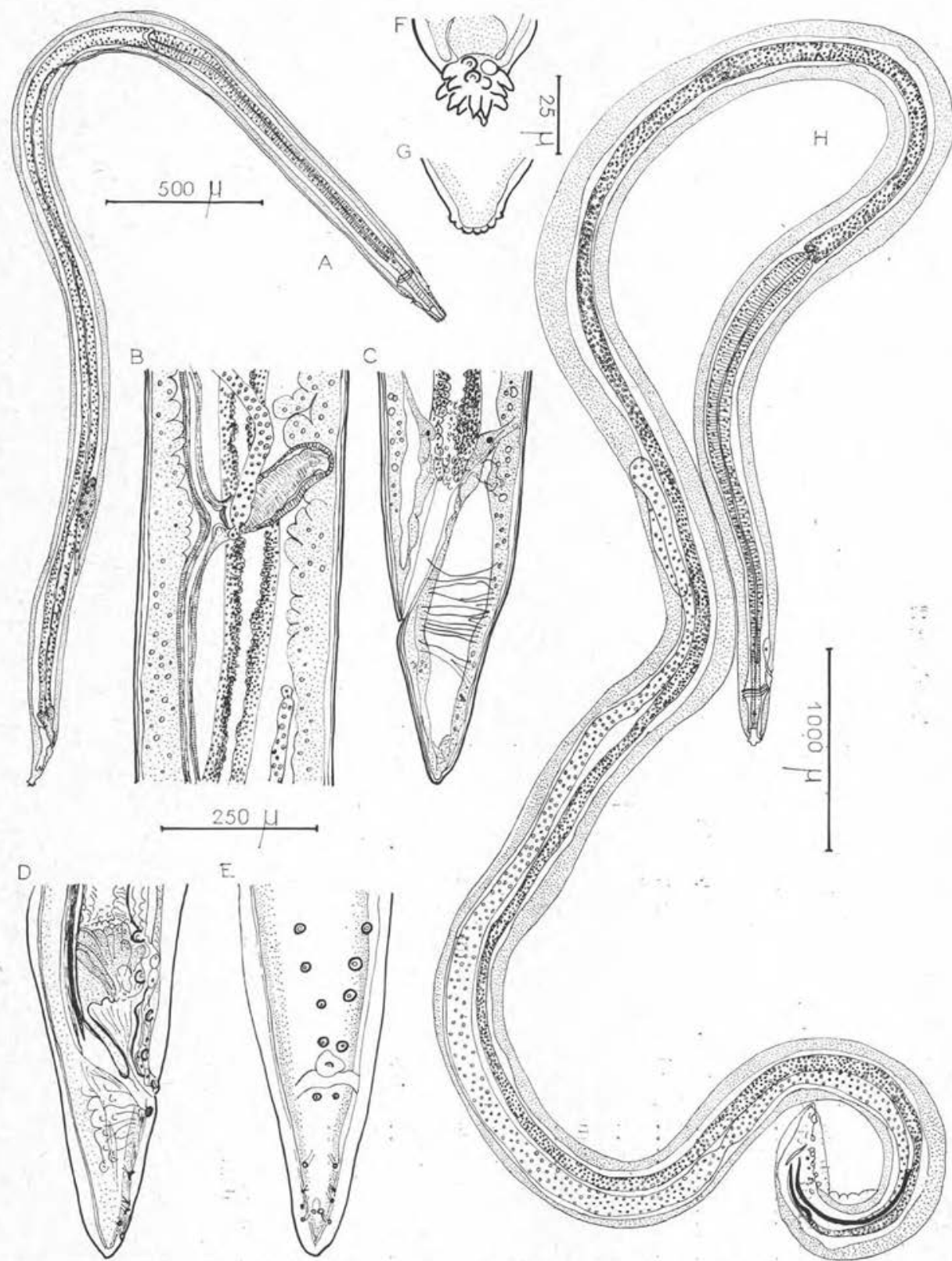

Fig. 4. - A : Larve du $3^{\text {e }}$ stade de cinq jours chez le Zygodontomys. - B: Larve du $4^{\circ}$ stade femelle, ovéjecteur, vue latérale. - C : Extrémité caudale, vue latérale. - D : Extrémité caudale d'une larve du $4^{e}$ stade mâle, vue latérale. - E : idem, vue ventrale. - H : Mâle de vingt jours. A: éch. $500 \mu$. B, C, D, E : éch. $250 \mu$. F, G: éch. $25 \mu$. H : éch. $1,000 \mu$ 


\begin{tabular}{|c|c|c|c|c|c|c|c|c|c|c|c|c|}
\hline \multirow{2}{*}{$\stackrel{m}{N}$} & of & के & 总 & 闹 & i & $\underset{\&}{\Phi}$ & 19 & 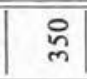 & \&) & 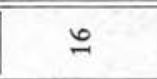 & $\left|\begin{array}{l}0 \\
\mid\end{array}\right|$ & \\
\hline & ${ }^{\circ}$ & तิ & $\mid \stackrel{\infty}{\infty}$ & |సి & f & 品 & 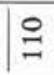 & 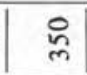 & 8 & $\infty$ & $\left|\frac{\mathrm{m}}{\mathrm{m}}\right|$ & శ్ఫণ \\
\hline \multirow{2}{*}{ 品 } & o+ & $\approx$ & |ః & | & \& & in & \& & 8 & \& & I & | & \\
\hline & ${ }_{0}$ & ส & 亲 & |ేి & 8 & \& & 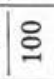 & 8 & 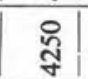 & $\infty$ & $\left|\begin{array}{l}\mid \\
\end{array}\right|$ & 丞导 \\
\hline \multirow{2}{*}{$\stackrel{\sim}{N}$} & o+ & $\approx$ & | & $\mid \stackrel{\circ}{\circ}$ & \& & $\frac{9}{6}$ & $\mid \cong$ & $\&$ & \&্ট্র| & \pm & $|\stackrel{\text { \& }}{\mid}|$ & \\
\hline & ${ }^{\circ}$ & ন & $\mid \stackrel{8}{g}$ & | & 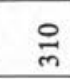 & 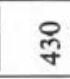 & | & D & ఫ్లె & فुे & $\mid \begin{array}{l}\text { \& } \\
\mid\end{array}$ & nొఝ \\
\hline \multirow{2}{*}{$\stackrel{\text { N }}{N}$} & o+ & 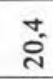 & | \& & $\mid \stackrel{\nexists}{~}$ & లి & \& & 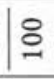 & \& & ठ & $\infty_{\infty}^{+}$ & $\mid \begin{array}{l}\text { d } \\
\text { | }\end{array}$ & \\
\hline & ro & \begin{tabular}{l}
$\infty$ \\
\multirow{\Xi}{*}{}
\end{tabular} & $\mid \stackrel{0}{\circ}$ & 18 & $\stackrel{\circ}{\circ}$ & : & 12 & 总 & $\begin{array}{l}0 \\
\text { a }\end{array}$ & $\underset{\sim}{\infty}$ & 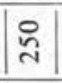 & จूँ \\
\hline \multirow{2}{*}{$\tilde{0}$} & ot & $\vec{\pi}$ & $\mid \begin{array}{l}\infty \\
\substack{\infty\\
}\end{array}$ & $1 ?$ & ๙ & $\underset{m}{R}$ & \& & : & $\begin{array}{l}\infty \\
\infty \\
m\end{array} \mid$ & $\infty$ & $\mid$ สి & \\
\hline & 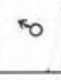 & 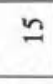 & $\mid \stackrel{8}{ }$ & $\mid \cong$ & $\stackrel{\text { iे }}{2}$ & 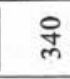 & $1 \approx$ & กิ & : & 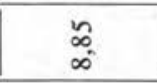 & $|\Xi|$ & ర్రల్ \\
\hline \multirow{2}{*}{ ป } & o+ & $\stackrel{\circ}{\circ}$ & 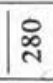 & $1 \cong$ & $\stackrel{\sim}{\circ}$ & 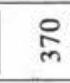 & $1 \infty$ & 字 & \&్d & 6 & 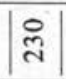 & \\
\hline & ${ }_{0}$ & 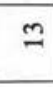 & | & |्ते & $\stackrel{4}{i}$ & $\stackrel{\circ}{\circ}$ & $\infty$ & $\stackrel{\circ}{\circ}$ & \&్సి & $b^{\circ}$ & $|\stackrel{\text { | }}{\mid}|$ & 응 \\
\hline \multirow{2}{*}{$\stackrel{\circ}{N}$} & of & $\simeq$ & |ని & $1 \tilde{0}$ & $\stackrel{\otimes}{\sim}$ & $\underset{ల}{\infty}$ & 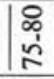 & กे & $\frac{8}{4}$ & ns & 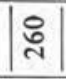 & \\
\hline & 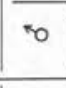 & $=$ & | & 12 & : & 品 & $R$ & 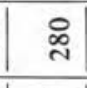 & ठ্ন| & $\frac{m}{5}$ & $\mid$ तి & \\
\hline$\frac{n}{N}$ & ro & $\stackrel{+}{n}$ & I & |워 & 品 & भ̊ & $1 \supseteqq$ & స్లి & 空 & r & $\mid$ |ू $\mid$ & \\
\hline \multirow{2}{*}{$\bar{u}$} & o+ & ○ & $1 \%$ & 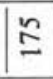 & $\stackrel{\circ}{\mathrm{N}}$ & 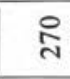 & $\mid 8$ & 品 & 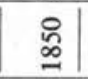 & 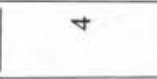 & $|\stackrel{0}{ }|$ & \\
\hline & o & $a$ & 1용 & 18 & ఏ & $\tilde{\text { ¿ }}$ & 18 & $\stackrel{\circ}{\pi}$ & 8 & ڤूे & $\left|\begin{array}{l}\infty \\
-1\end{array}\right|$ & \\
\hline 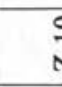 & e & $\underset{6}{\infty}$ & |ః & In & $\stackrel{\circ}{\mathrm{N}}$ & $\stackrel{\text {. }}{\circ}$ & 18 & ఫั & 8 & $N$ & $|\stackrel{\infty}{\infty}|$ & \\
\hline i & jo & $\stackrel{n}{i}$ & | & ఫิ & פ & in & IR & 导 & 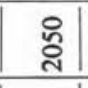 & $\cong$ & in & \\
\hline 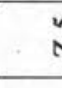 & 5 & $\stackrel{n}{m}$ & 요 & 18 & 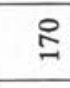 & i & 18 & $气$ & $\stackrel{\square}{\circ}$ & $\stackrel{\infty}{0}$ & $\cong$ & \\
\hline 薴 & & 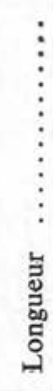 & 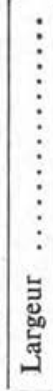 & 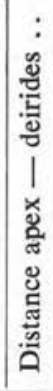 & 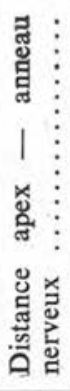 & 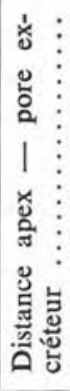 & 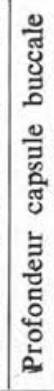 & 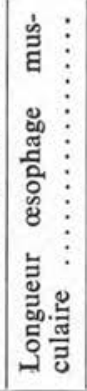 & 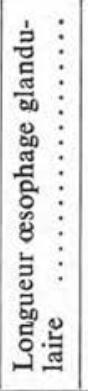 & 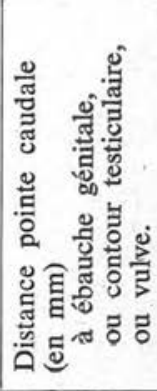 & 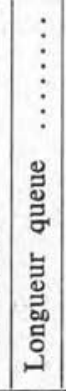 & 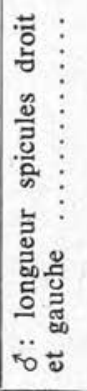 \\
\hline
\end{tabular}


Pourcentages des larves et des adultes retrouvés par rapport au nombre de larves infestantes:

$\begin{array}{ll}\text { Zygodontomys } & 54 \% \\ \text { Calomys } & 39 \% \\ \text { Oryzomys } & 60 \%\end{array}$

1) Evolution chez Zygodontomys. $24 \mathrm{~h}$ après l'infestation chez l'hôte définitif, la larve du troisième stade n'a pas évolué, ses dimensions sont voisines de celles de la larve encapsulée âgée de trente jours chez la Forficule. Cependant, l'évolution du troisième stade larvaire va se poursuivre chez l'hôte définitif jusqu'aux environs du dixième et du onzième jour.

Au cinquième jour (fig. 4 A), la larve atteint $3750 \mu$ et l'œsophage glandulaire n'a pas grandi. C'est à partir du cinquième jour que cette larve va doubler de longueur jusqu'à la troisième mue.

Les principales modifications qui interviennent après cette troisième mue sont : la perte des pointes caudales qui ornaient l'extrémité postérieure dilatée en mamelon des larves du troisième stade (fig. $4 \mathrm{G}$ ) ; - la réduction de l'aile latérale, toujours visible, mais devenue très fine ; - la différenciation déjà ébauchée à la fin du troisième stade des sexes mâles et femelles (fig. 4 B, C et 4 D, E) ; - enfin, une brusque augmentation de la taille, qui intervient à la fois chez les larves mâles et chez les larves du quatrième stade, femelles.

L'évolution des larves mâles et femelles du quatrième stade se prolonge jusqu'à la dernière mue, qui débute vers le dix-huitième jour du développement chez le Zygodontomys. Le mâle adulte de vingt jours mesure $14,85 \mathrm{~mm}$ de long sur $360 \mu$ de large. La longueur de la femelle adulte est de $20 \mathrm{~mm}, 400 \mu$ de large. L'aile latérale a disparu.

2) Comparaison de l'évolution chez Zygodontomys, chez Calomys et ORYzomYs.

Nous résumons dans le tableau 2 les dimensions des différents stades larvaires en indiquant par la lettre $\mathbf{Z}$ les parasites récoltés chez le Zygodontomys, par la lettre $\mathbf{C}$ ceux recueillis chez le Calomys, et par O ceux récoltés chez l'Oryzomys.

La longueur totale du corps est en $\mathrm{mm}$, ainsi que la distance séparant l'ébauche génitale de la pointe caudale ; toutes les autres dimensions sont en $\mu$.

Le tableau, et le graphique établi d'après les longueurs totales du corps en fonction de la durée de développement chez l'hôte définitif, montrent que l'évolution est comparable chez les différents Cricetidae, tant dans la croissance des différents stades, que dans l'apparition des troisième et quatrième mues.

3) Infestation de Muridae africains.

- Un Hybomys univittatus (Peters) infesté avec cinq larves du troisième stade de Protospirura numidica, et autopsié au bout de vingt-cinq jours, héberge dans son estomac trois femelles, dont les dimensions $(27,29$ et $30 \mathrm{~mm})$ sont inférieures à celles obtenues $(35 \mathrm{~mm})$ pour une durée identique de développement chez le Zygodontomys.

- Un Hybomys infesté par six larves du quatrième stade de douze jours, ayant effectué la troisième mue chez un Zygodontomys, est autopsié vingt-huit jours après ; 
son estomac héberge deux mâles longs de $16 \mathrm{~mm}$ et une femelle longue de $33 \mathrm{~mm}$. Ces dimensions sont inférieures à celles d'adultes mâles et femelles qui se sont développés entièrement chez Zygodontomys durant une période plus courte. Les longueurs des spicules chez deux mâles recueillis chez l'Hybomys sont : $1050 \mu$ et $440 \mu, 1140 \mu$ et $460 \mu$.

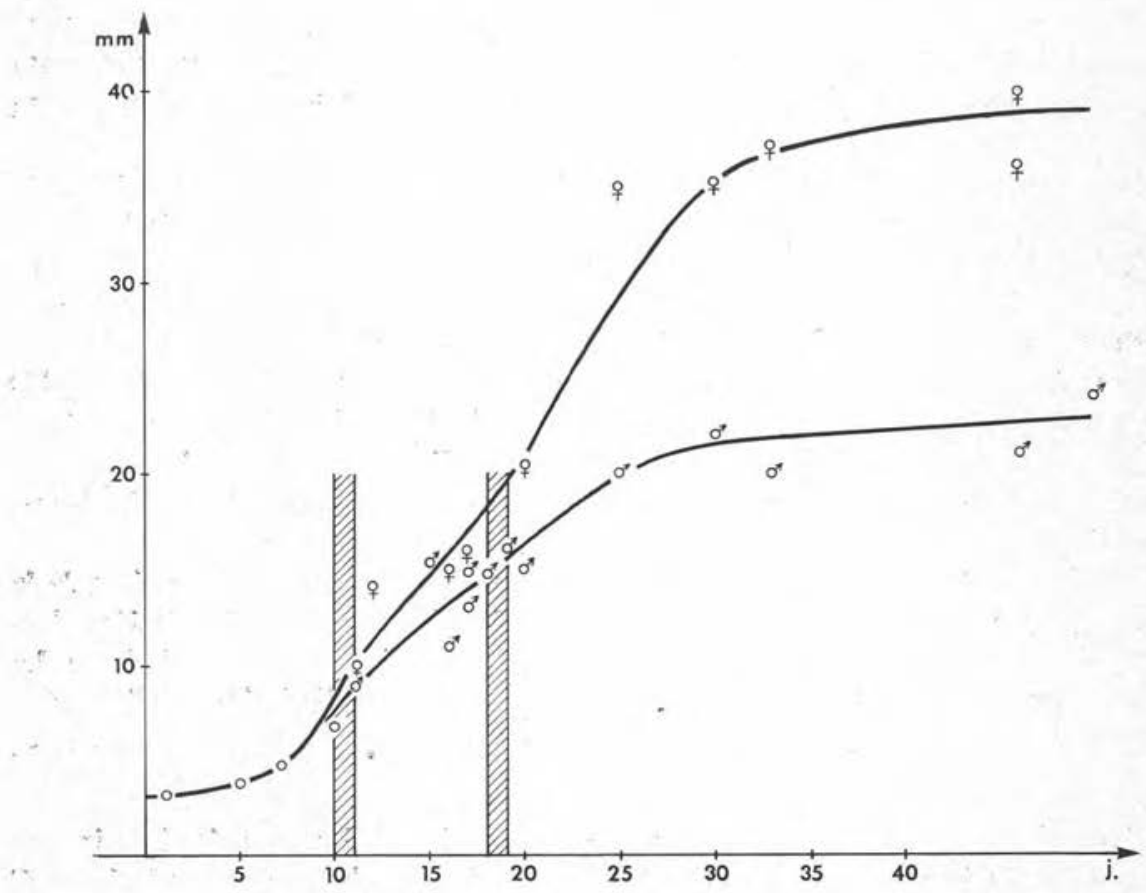

Fig. 5. - Graphique. Croissance de P. n. criceticola : en abscisse, durée de développement' en jours ; en ordonněe, longueur des larves et des adultes en $\mathrm{mm}$.

\section{Discussion.}

En Amérique du Nord, le cycle évolutif de Protospirura numidica criceticola a été réalisé en 1963 par J. R. Crook et A. W Grundmann chez de nombreuses espèces de Coléoptères et d'Orthoptères recueillis dans l'habitat naturel du Peromyscus maniculatus, ainsi que chez des Insectes de laboratoire. L'évolution étudiée surtout d'après la sroissance des capsules à $25^{\circ} \mathrm{C}$ et des infestations réalisées après vingt, trente et quarante jours de développément chez Eleodes tuberculata palustris montrent que c'est entre trente et quarante jours que la larve devient infestante pour l'hôte définitif : Peromyscus.

Chez les Forficules récoltées et infestées au Brésil, pour une température très voisine, le troisième stade est obtenu le vingt-huitième jour, et la durée du développement chez l'hôte définitif est comparable puisque, chez Peromyscus, les premiers œufs 
sont retrouvés dans les fèces 34 jours après infestation et 33 jours suffisent chez le Zygodontomys pour obtenir les premiers œufs embryonnés.

Le développement étudié chez la Forficule Doru lineare puis chez le Zygodontomys présente une réduction progressive de la durée des stades larvaires : quatorze jours sont en effet nécessaires à $25^{\circ} \mathrm{C}$ après l'infestation de l'hôte intermédiaire pour obtenir la première mue ; douze jours après survient la seconde ; dix jours suffisent au développement du troisième stade larvaire chez l'hôte définitif et l'obtention de la troisième mue. La durée du quatrième stade est de huit jours. La croissance du mâle se termine-entre le vingt-cinquième et le trentième jour, après l'infestation chez l'hôte définitif. Les femelles présentent dans l'ovéjecteur les œufs complètement embryonnés vèrs le trente-troisième jour ; la croissance devient alors très lente.

Au Brésil, la présence de Protospirura numidica criceticola presque exclusivement chez le Rongeur Cricetidae Zygodontomys ne semble due qu'aux conditions écologiques. L'infestation expérimentale d'autres Rongeurs Cricetidae de la même région, Calomys callosus et Oryzomys nigripes ne montre pas de différences significatives dans le développement du parasite.

\section{Bibliographie}

Butler (J. M.) et Grundmann (A. W.), 1954. - The intestinal helminths of the coyote Canis latrans, Say, in Utah. J. Parasitol., 40, (4), p. 440-443.

CHITwood (B. G.), 1938. - The status of Protospirura vs. Mastophorus, with consideration of the species of these genera. Livro Jub. Travassos, p. 115-118, tab. 1-2, fig. 1-22.

Crook (J. R.) et Grundmann (A. W.), 1964. - The life history of Protospirura numidica Seurat 1914 (Nematoda: Spiruroidea). Proc. Helm. Soc. Washington, 31, (2), p. 225-229, fig. 1-4.

GrundmanN (A. W.), 1957. - Nematode parasites of Mammals of the great salt lake desert of Utah. J. Parasitol., 43, (4), p. 105-112.

- et Frandsen (J.C.), 1960. - Definitive host relationships of the helminth parasites of the deer mouse Peromyscus maniculatus in the Bonneville Basin of Utah. Ibid., 46, p. 673-677.

Seurat (L.-G.), 1914. - Sur un nouveau spiroptère du Chat ganté. C.R. Soc. Biol., Paris, 77 , p. $344-347$, fig. $1-5$.

—, 1916. - Sur l'habitat normal et les affinités de Protospirura numidica, Seur. Ibid., 79, p. 143-146, fig. 1-5.

Skrjabin (K. I.) et Sobolev (A. A.), 1963. - Osnovi Nematodologi 11. Publ. Acad. Sc. U.R.S.S., Moscou, p. 1-5.11, fig. 1-290 (En Russe). 


\section{Addendum}

Nous avons pris connaissance, durant l'impression de ce travail, de la publication de W. G. DYer \& O.W. Olsen, 1967. Biology of Mastophorus numidica (Seurat, 1914), Read and Milleman, 1953 (Nematoda Spiruridae) with description of juvenile stages. Proc. Helm. Soc. Washington, 34 (1), p. 98-103, fig. 1-13. Ces auteurs précisent le cycle réalisé par Crook \& Grundmann en Amérique du Nord, et décrivent les stades larvaires de ce Spiruride chez trois espèces d'Insectes: Melanoplus femur rubrum, Eleodes obsedata, Acheta domestica, puis chez l'hôte définitif: Peromyscus maniculatus. Nos données: morphologie, dimensions, durée de développement du parasite, concordent avec leurs résultats. 